\title{
The Effect of Interferon on Relapse Rate in Multiple Sclerosis
}

K.S.Moselhy, M.K.Fahem, S.M.Kassem, and R.M.Abd El-Fatah

Neurology Dept., Faculty of Medicine, Benha Univ., Benha, Egypt

E-Mail:Rana11@gmail.com

\begin{abstract}
Multiple sclerosis (MS) is a chronic inflammatory demyelinating central nervous system disease that typically strikes young adults, especially women. The pathobiology of MS includes inflammatory and neurodegenerative mechanisms that affect both white and gray matter. This was a retrospective comparative study conducted on 50 patients with Multiple Sclerosis (MS); to assess EDSS score (Expanded Disability Status Scale) and Relapse Rate of M.S patients after one year of receiving Interferon. This is Retrospective, randomized study included Fifty Egyptian patients with relapsing RRMS were recruited from Neurology department of Maadi military hospital and Ain Shams university hospital, as well as outpatient clinics. The included patients were all receiving medications in the form of disease modifying therapies (DMTs) (Immunomodulators). Results; the mean age of all patients was (32.5 \pm 3.6$)$ years. Regarding gender of the patients, the majority $(66 \%)$ of patients were males; while $(34 \%)$ were females. Comparative study between the 3 groups revealed Non-significant difference as regards age and sex of the patients $(p>0.05)$. highly significant decrease in 1-year EDSS score and relapse rate, in Rebif® then Betaferon ${ }^{\circledR}$ group; compared to Avonex ${ }^{\circledR}$ group ( $\mathrm{p}<0.01$ respectively). Spearman's correlation analysis shows that; Age, and age at onset, had a highly significant positive correlation with 1 -year relapse rate $(\mathrm{p}<0.05$ respectively).
\end{abstract}

Keywords: Interferon, Relapse, Multiple Sclerosis, MS.

\section{Introduction}

Multiple sclerosis (MS) is a chronic inflammatory demyelinating central nervous system disease that typically strikes young adults, especially women. The pathobiology of MS includes inflammatory and neurodegenerative mechanisms that affect both white and gray matter [1].

The worldwide prevalence of MS is estimated at $50: 100,000$, with $2.7-5 \%(1.35-2.5: 100,000)$ of these cases occurring before the age of 18 years, and about $0.2-0.7 \%(0.4-1.4: 100,000)$ occurring during infancy and early childhood [2].

MS results from a complex interaction between environmental factors, the genetic background of the individual that defines individual susceptibility, and the immunological and physiological setting of the individual. so, clinical manifestations are variable and include episodes of neurological dysfunction, such as numbness, weakness, diplopia and vision loss [3].

Several clinical courses are described in MS. About $85 \%$ of people are diagnosed with relapsing remitting MS (RRMS). About $75 \%$ of RRMS patients will go on to develop secondary progressive MS (SPMS), this progression to SPMS will occur within 10-15 years of the initial diagnosis in $50 \%$ of patients. For about $10-15 \%$ of people with MS, their disease is associated with progressive disability from the outset, so-calledprimary progressive MS (PPMS) [4].

The 2010 revision of the McDonald Criteria defining dissemination in time (DIT) and space (DIS) has simplified the diagnostic criteria of MS and resulted in earlier and more reliable diagnosis [5]. Dissemination of disease in space and time proved by either clinical, paraclinical or laboratory assessments is an essential diagnostic criterion for MS. Differential diagnosis for the clinical presentation must be considered and excluded before MS can be diagnosed.
Cranial MRI shows lesions in at least $95 \%$ of MS patients and plays a key role in diagnosis and followup. [5].

The extent of disability in MS may be measured using a variety of scales; the most commonly used is the Expanded Disability Status Scale (EDSS), an observer-rated scale which evaluates a patient's level of functional impairment on a scale of $0-10$ (0 describing no disability and 10 describing death due to MS). Although one of the characteristics of MS is its unpredictability, the disease pattern in the first 5 years may be prognostic for further disability and disease progression. [6].

The goals of management of MS are to (1) reduce the frequency and severity of relapses and slow the progression of the disease and (2) manage the symptoms associated with MS in order to maintain quality of life. Management of MS requires the involvement of a multidisciplinary team (MDT) of healthcare professionals, using both pharmacological and non-pharmacological modalities of treatment. [7].

Corticosteroids are typically used to manage acute symptoms or relapses in adults and also in children with MS. Today, most important first-line treatment option for RRMS patientsstill self-injectable diseasemodifying therapies as interferon beta based drugs (IFN beta) or Glatiramer acetate (GA). Both classes of drugs are well proven to reduce disease activity in RRMS and have a good safety profile. However, about one third of RRMS patients show an insufficient response to these drugs [8].

This study aimed to assess EDSS score (Expanded Disability Status Scale) and Relapse Rate of Relapsingremitting multiple sclerosis(RRMS) patients after one year of receiving Interferon therapy. 


\section{Patients and methods}

This was a retrospective comparative study conducted on 50 patients with Multiple Sclerosis (MS);to assess EDSS score (Expanded Disability Status Scale) and Relapse Rate of M.S patients after one year of receiving Interferon.

A total of 50 Egyptian patients with relapsing remitting multiple sclerosis RRMS enrolled in the study.

Patient's age ranged from 20 to 55 years with EDSS score below 6 .

All patients were diagnosed as clinically definite multiple sclerosis (CDMS) according to the 2010 revised McDonald's criteria [5].

\section{Inclusion criteria}

1- All patients were diagnosed as clinically definite multiple sclerosis (CDMS) according to the 2010 revised McDonald's criteria

2- Patient's age ranged from 20 - 55 years.

3- EDSS score $\leq 6$.

4- M.S patients received Interferons.

\section{Exclusion criteria}

1- Patients with Primary Progressive Multiple Sclerosis (PPMS).

2- Secondary Progressive Multiple Sclerosis (SPMS) patients.

3- Neuromyelitis Optica (NMO) patients.

4- Patients with chronic co-morbidities such as (hypertension \& diabetes mellitus \& etc.)
All patients subjected to: Full history taking, general medical examination and Neurological examination. And EDSS scale

Patients were randomized according to type of Interferon used :

- IFN及-1a (Avonex®):30 $\mu \mathrm{g} \quad$ Once weekly intramuscularly.

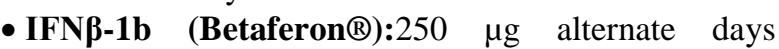
subcutaneously.

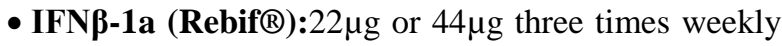
subcutaneously.

\section{Statistical analysi}

Data entry, processing and statistical analysis was carried out using MedCalc ver. 18.2.1 (MedCalc, Ostend, Belgium). Tests of significance (KruskalWallis, Chi square and Wilcoxon's tests, factorial ANOVA, logistic regression analysis, Spearman's correlation and ROC Curve analysis) were used. Data were presented and suitable analysis was done according to the type of data (parametric and nonparametric) obtained for each variable. P-values less than $0.05(5 \%)$ was considered to be statistically significant.

\section{Results}

The mean age of all patients was $(32.5 \pm 3.6)$ years. Regarding gender of the patients, the majority $(66 \%)$ of patients were males; while $(34 \%)$ were females Table (1).

Table (1) Socio-demographic data among 50 RRMS patients.

\begin{tabular}{lcc}
\hline Variables & & Frequency (\%) \\
\hline Age (years) & Female & $32.5 \pm 3.6^{*}$ \\
Gender & Male & $33(66 \%)$ \\
\hline \multicolumn{3}{c}{} \\
& $*$ Mean \pm SD.
\end{tabular}

Comparative study between the 3 groups revealed non-significant difference as regards all baseline clinical and neurological data $(p>0.05)$ Table $(2)$

Table (2) Comparison between the 3 groups as regards basic clinical and neurological data using Kruskal-Wallis and Chi square tests.

\begin{tabular}{|c|c|c|c|c|c|}
\hline \multirow{2}{*}{\multicolumn{2}{|c|}{ Variable }} & $\begin{array}{c}\text { Avonex@ group } \\
(17)\end{array}$ & $\begin{array}{c}\text { Betaferon }{ }^{\circledR} \text { group } \\
(16)\end{array}$ & $\begin{array}{c}\text { Rebif®group } \\
\text { (17) }\end{array}$ & $\begin{array}{c}\text { Kruskal- } \\
\text { Wallis } \\
\text { test }\end{array}$ \\
\hline & & Median (IQR) & Median (IQR) & Median (IQR) & P value \\
\hline \multicolumn{2}{|l|}{ Age at onset (year) } & $29(26.7-31)$ & $29.5(27-31)$ & $27(26-29.2)$ & $=0.1914$ \\
\hline \multicolumn{2}{|l|}{ MS duration(year) } & $4(3-5.2)$ & $4(3-5)$ & $3(2-5)$ & $=0.5033$ \\
\hline \multicolumn{2}{|l|}{ Relapses in previous year } & $2(1-2)$ & $2(1-2.5)$ & $3(2-3)$ & $=0.1403$ \\
\hline \multicolumn{2}{|l|}{ EDSS score } & $4(3-4)$ & $4(3.5-4)$ & $4(4-4.1)$ & $=0.2064$ \\
\hline \multicolumn{2}{|l|}{ Number of presenting symptoms } & $1(0-3)$ & $1(0-3)$ & $3(0.7-3)$ & $=0.6118$ \\
\hline Variable & & $\begin{array}{c}\text { Avonex } ₫ \text { group } \\
\text { (17) }\end{array}$ & $\begin{array}{c}\text { Betaferon }{ }^{\circledR} \text { group } \\
\text { (16) }\end{array}$ & $\begin{array}{c}\text { Rebif®group } \\
\text { (17) }\end{array}$ & $\begin{array}{c}\text { Chi square } \\
\text { test } \\
\text { P value }\end{array}$ \\
\hline - Motor weakness & $+\mathrm{ve}$ & $8(47.1 \%)$ & $6(37.5 \%)$ & $12(70.6 \%)$ & $=0.1446$ \\
\hline - Sensory loss & $+\mathrm{ve}$ & $9(52.9 \%)$ & $9(56.2 \%)$ & $10(58.8 \%)$ & $=0.9418$ \\
\hline
\end{tabular}


Table (2) Continue

- Cranial $\mathrm{N}$ affection

- Autonomic symptoms $+\mathrm{ve}$ $6(35.3 \%)$ $2(11.8 \%)$

*Percentage of Column Total.

Comparative study between baseline and 1-year assessments revealed; highly significant decrease in 1- year EDSS and relapses assessments in all 3 drug groups ( $\mathrm{p}<0.01$ respectively) Table (3).

Table (3) Comparison between allpatients as regards serial EDSS and relapses assessments.

\begin{tabular}{lccc}
\hline \multirow{2}{*}{ Avonex® group } & $\begin{array}{c}\text { Baseline } \\
\text { measurement }\end{array}$ & $\begin{array}{c}\text { 1-year } \\
\text { measurement }\end{array}$ & Wilcoxon's test \\
\cline { 2 - 4 } & Median (IQR) & Median (IQR) & P value \\
\hline EDSS score (1-year) & $4(3-4)$ & $3(1-3)$ & $<0.0001^{* *}$ \\
Relapse rate (1-year) & $2(1-2)$ & $1(1-1.2)$ & $=0.001^{* * *}$ \\
& Baseline & $1-$ year & Wilcoxon's test \\
Betaferon® group & measurement & measurement & P value \\
EDSS score (1-year) & Median (IQR) & Median (IQR) & $<0.0001^{* *}$ \\
Relapse rate (1-year) & $4(3.5-4)$ & $1(1-2)$ & $=0.001^{* *}$ \\
& $2(1-2.5)$ & $0(0-1)$ & Wilcoxon's test \\
Rebif® group & Baseline & 1 -year & P value \\
& measurement & measurement & $<0.0001^{* *}$ \\
EDSS score (1-year) & Median (IQR) & Median $(\mathrm{IQR})$ & $<0.0001^{* *}$ \\
Relapse rate (1-year) & $4(4-4.1)$ & $1(0.8-1)$ & $0(0-0.2)$ \\
\hline
\end{tabular}

Spearman's correlation analysis shows that; all baseline clinical / neurological factor had a non- significant correlation with 1-year EDSS score ( $\mathrm{p}>$ 0.05 respectively) Table (4).

Table (4) Spearman's correlation analysis for baseline clinical/ neurological Factors associated with1-year EDSS score.

\begin{tabular}{lcc}
\hline \multirow{2}{*}{ Associated Factor } & \multicolumn{2}{c}{ 1-year EDSS score } \\
\cline { 2 - 3 } & rho & P \\
\hline Age (years) & 0.126 & $=0.3832$ \\
Age at onset (year) & 0.198 & $=0.1679$ \\
MS duration(year) & 0.0130 & $=0.9285$ \\
Relapses in previous year & -0.200 & $=0.1641$ \\
EDSS score & 0.206 & $=0.1521$ \\
Number of presenting symptoms & -0.0256 & $=0.8597$ \\
\hline
\end{tabular}

rho: Spearman's rho (correlation coefficient).

Spearman's correlation analysis shows that; age, and age at onset,had a highly significant positive correlation with 1-year relapse rate $(\mathrm{p}<0.05$ respectively) Table (5).

Table (5) Spearman's correlation analysis for baseline clinical / neurological Factors associated with1-year relapse rate.

\begin{tabular}{lcc}
\hline \multirow{2}{*}{ Associated Factor } & \multicolumn{2}{c}{ 1-year relapse rate } \\
\cline { 2 - 3 } & rho & P \\
\hline Age (years) & 0.392 & $=0.0048^{* *}$ \\
Age at onset (year) & 0.285 & $=0.045^{*}$ \\
MS duration(year) & 0.219 & $=0.1257$ \\
Relapses in previous year & 0.111 & $=0.4429$ \\
EDSS score & -0.0436 & $=0.7636$ \\
Number of presenting symptoms & 0.0101 & $=0.9445$ \\
\hline
\end{tabular}

rho Spearman's rho (correlation coefficient).

By using ROC-curve analysis, Rebif® drug with fair (77.9\%) accuracy, sensitivity $=58.8 \%$ and treatment predicted decrease in 1-year EDSS score, specificity $=87.8 \%(\mathrm{p}<0.01)$ Fig $(1)$ 


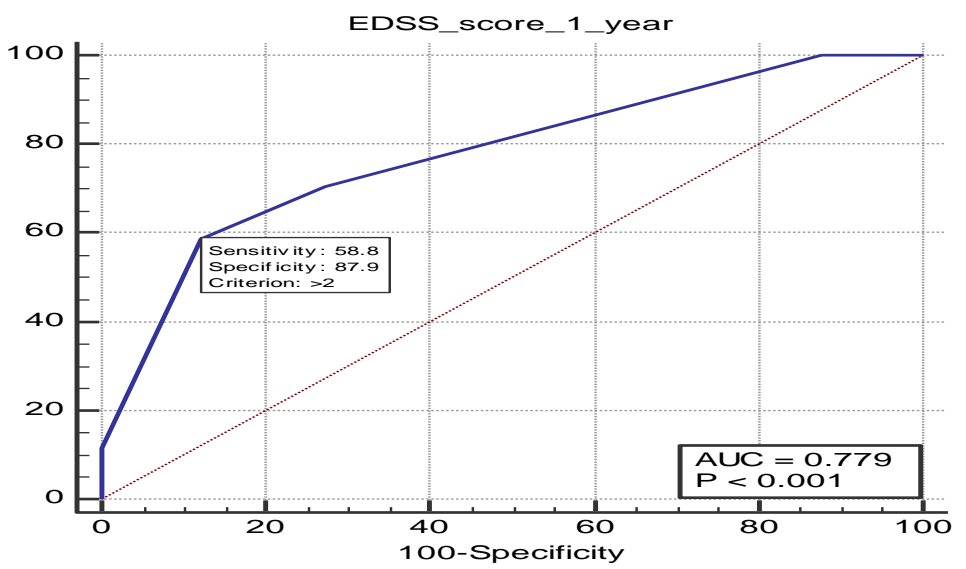

Fig (1) ROC curve of Rebif® (decrease in 1-year EDSS score).

By using ROC-curve analysis, Betaferon ${ }^{\circledR}$ drug treatment predicted decrease in 1-year EDSS score, with fair $(75.8 \%)$ accuracy, sensitivity $=82 \%$ and specificity $=54.5 \%(\mathrm{p}<0.01)$ Fig $(2)$.

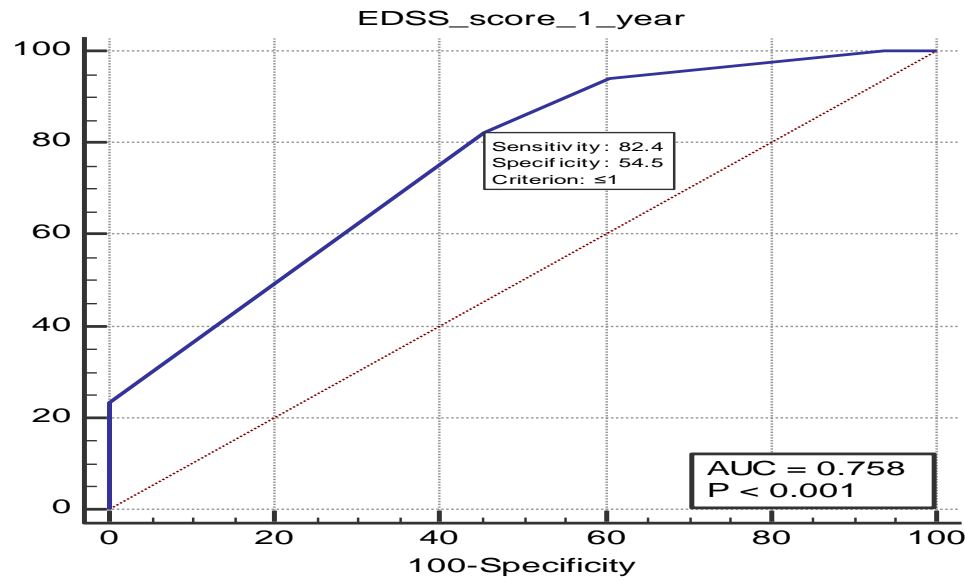

Fig (2) ROC curve of Betaferon ${ }^{\circledR}$ (decrease in 1-year EDSS score).

By using ROC-curve analysis, Betaferon $®$ drug treatment predicted decrease in 1-year relapse rate, with fair (74.5\%) accuracy, sensitivity $=76 \%$ and specificity $=69.7 \%(\mathrm{p}<0.01)$ Fig $(3)$.

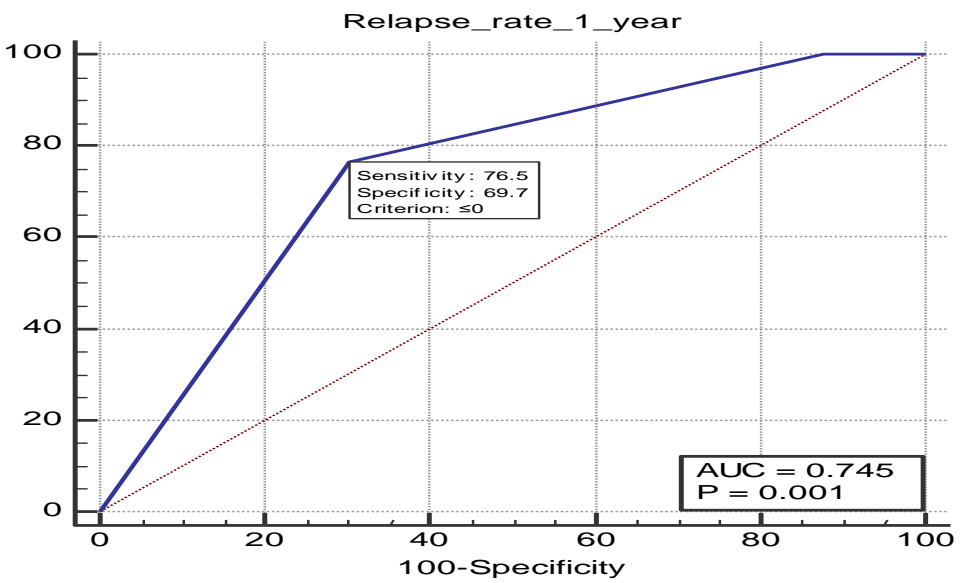

Fig (3) ROC curve of Betaferon $®($ decrease in 1-year relapse rate). 


\section{Discussion}

We found that; the mean age of all patients was $(32.5 \pm 3.6)$ years. Regarding gender of the patients, the majority $(66 \%)$ of patients were males; while $(34 \%)$ were females, which came in agreement with [9], [10].

Mazdehet al., reported that,Ninety patients were included in this study with anaverage age of $31.11 \pm$ 8.62 with a minimum of 14 and maximum age of $50.72(80 \%)$ were female and $18(20 \%)$ were male [10].

Comparative study between the 3 groups revealed non-significant difference as regards age and sex of the patients $(\mathrm{p}>0.05)$., which came in agreement with previous studied. Blaşa et al. reported that, although the patients were not randomized to treatment, no significant differences were observed among the three groupswith regard to age, gender, age at disease [11].

Mazdeh et al., reported that,Comparative study of the average First and Final EDSS, using the paired sample t-test, showed asignificant statistical difference in all three groups [10].

Comparative study between the 3 groups revealed; highly significant decrease in 1-year EDSS score and relapse rate, in Rebif $\AA$ then Betaferon ${ }^{\circledR}$ group; compared to Avonex ${ }^{\circledR}$ group ( $\mathrm{p}<0.01$ respectively)., which came in agreement with [10], [12]

Mazdeh et al., 2010 reported that ,Rebif caused a significant statistical difference in the average relapse rate before and after treatment $(\mathrm{P}<0.05)$, and compared to the other two drugs, it had a greater effect on decrease in relapse $(62.5 \%)$ [10]

Mokhber et al. 2015 reported that,within each group, participants who received $\operatorname{Rebif}(\mathrm{P}=0.001)$ and Betaferon $(\mathrm{P}=0.028)$ experienced an improvement in their disability; however, those who received Avonex did not $(\mathrm{P}=0.548)$ [12]

Spearman's correlation analysis shows that; age, and age at onset, had a highly significant positive correlation with 1 -year relapse rate $(\mathrm{p}<0.05$ respectively), which came in disagreement with Madjdinasab et al. , reported that, the age of the patients did not have a role in the outcome of the treatment. Each of the three types of interferon (Avonex , Rebif and Betaferon) has the same effects equivalent to a $30 \%$ relapse reduction in MS attacks in the patients [13].

Multiple regression analysis shows that; after applying (Forward method) and entering some predictor variables; the increase in baseline EDSS score; had an independent effect on increasing 1-year EDSS score; with significant statistical difference $(\mathrm{p}=$ 0.0019), which came in agreement with Shahtaheri et al., reported that, the model calculated the following outcomes: average number of years spent in EDSS 0.05.5; average number of years spent relapse-free; quality-adjusted life-years (QALYs); 10-year costs; and incremental cost-effectiveness ratios (ICERs) comparing Cinnovex with Avonex. Model parameters varied in sensitivity analyses. The results showed that the ICER was relatively insensitive to changes in QOL variables in Cinnovex and Anovex at 6-7.5 and 3-5.5 scores [14].

Multiple regression analysis shows that; after applying (Forward method) and entering some predictor variables; the increase in relapses in previous year and Avonex ${ }^{\circledR}$ usage; had an independent effect on increasing 1-year relapse rate; with significant statistical difference $(\mathrm{p}<0.05$ respectively)., which came in agreement with Minagar, 2013 reported that,treatment of MS patients with IFN- $\beta 1$ a decreased relapse rate by $18 \%$ for the total group and $32 \%$ for those who finished the 24-month medication course of the trial [15].

By using ROC-curve analysis, Rebif® drug treatment predicted decrease in 1-year EDSS score, with fair $(77.9 \%)$ accuracy, sensitivity $=58.8 \%$ and specificity $=87.8 \% \quad(\mathrm{p}<0.01)$, which came in agreement with Minagar, 2013 reported that,based on the results of this comparative trial ,after 24 weeks, $74.9 \%$ of patients who were treated with IFN- $\beta 1$ a 44 $\mu \mathrm{g}$ three times weekly stayed relapse-free compared with $63.3 \%$ of those who were treated with IFN- $\beta$ 1a 30 $\mu \mathrm{g}$ once weekly. The odds ratio for staying free from relapse was $1.9(95 \% \mathrm{CI}, 1.3-2.6 ; P=0.0005)$ at 24 weeks and $1.5(95 \% \mathrm{CI}, 1.1$ to $2.1 ; P=0.009)$ at 48 weeks in favor of IFN- $\beta 1 \mathrm{~b} 44 \mu \mathrm{g}$ three times weekly [15].

By using ROC-curve analysis, Betaferon ${ }^{\circledR}$ drug treatment predicted decrease in 1-year EDSS score, with fair $(75.8 \%)$ accuracy, sensitivity $=82 \%$ and specificity $=54.5 \%(\mathrm{p}<0.01)$, which came in agreement with Mokhber et al.,2015 reported that, Betaferon improved the Overall QOL $(\mathrm{P}=0.21)$ and Emotional wellbeing $(\mathrm{P}=0.034)$ subscales better than the other two treatments [12].

By using ROC-curve analysis, Avonex ${ }^{\circledR}$ drug treatment showed non-significant predictive values in discrimination of patients with decrease in 1-year EDSS score from patients without $(p>0.05)$, which came in agreement with [14], [15]

Minagar, 2013 reported that ,intramuscularly once weekly) or placebo, for 24 months. The patients' EDSS scores were between 1.0 and 3.5, and each patient had experienced at least two relapses in the three years prior to study initiation. The clinical trial was discontinued earlier than originally designed, and only $57 \%$ of patients finished the two-year length of the study. Many conclusions were drawn based on this group of the patients who were treated with two years of IFN- $\beta 1 \mathrm{a}$ and not the whole group which would have included all participants. After 24months, treatment of MS patients with IFN- $\beta 1$ a (Avonex) was associated with an effect on the primary end point of the trial, the progression rate of at least 1.0 point on theEDSS compared to placebo [15].

Shahtaheri et al., reported that,the results showed that the ICER was relatively insensitive to changes in QOL variables in Cinnovex and Anovex at 6-7.5 and 35.5 scores. On the other hand, the changes in other 
variables had no significant effects on the final output probability in the two strategies [14].

By using ROC-curve analysis, Rebif® drug treatment predicted decrease in 1-year relapse rate, with good (84\%) accuracy, sensitivity $=94 \%$ and specificity $=66 \%(\mathrm{p}<0.01)$, which came in agreement with Madjdinasab et al.,2015 reported that,studies have shown that Rebif has a beneficial effect on the rate of relapse and lesions from MRI in MS patient's brain compared to placebo.A study of the efficacy of three drugs of Avonex, Rebif and Betaferon in reducing relapses and EDSS did not find significant differences in reducing relapses and EDSS among patients receiving these three drug [13].

By using ROC-curve analysis, Betaferon ${ }^{\circledR}$ drug treatment predicted decrease in 1-year relapse rate, with fair $(74.5 \%)$ accuracy, sensitivity $=76 \%$ and specificity $=69.7 \% \quad(\mathrm{p}<0.01)$, which came in agreement with Minagar 2013 reported that ,patients who were treated with the higher dose of IFN- $\beta 1 \mathrm{~b}$ compared to those treated with the lower dose showed more decrease in their clinical relapse rate $(P=0.0086)$ [15].

\section{Conclusion}

This comparative trial of the three IFN-b formulations provides compelling evidence that treatment regimen has an effect on efficacy. The data further support the contention that the three IFN-b formulations are useful tools to prevent the relapses and to slow down the neurological deterioration in RRMS with Betaferon and Rebif demonstrating more effectiveness than Avonex. These results have important implications for the recommendation and selection of disease modifying therapies in the management of RRMS.

\section{References}

[1] D. M. Wingerchuk and J. L. Carter, "Multiple sclerosis: current and emerging disease-modifying therapies and treatment strategies," in Mayo Clinic Proceedings, Vol.89(2), PP.225-240,2014.

[2] M. Lysenko, "Functional MRI Activation of Inhibitory Control in Adolescents and Young Adults with Multiple Sclerosis,", Vol.1(2), PP.65-87, 2013.

[3] B. K. Paap, M. Hecker, D. Koczan, and U. K. Zettl, "Molecular biomarkers in multiple sclerosis," J. Clin. Cell. Immunol. S, Vol.10(4), PP.34-65, 2013.

[4] L. M. Samkoff, "Immunomodulatory Agents for relapsing-Remitting Multiple Sclerosis." Giesser
BS. Primer on Multiple Sclerosis. Oxfored University Press, Vol.1(3), pp 112-154, 2011.

[5] C. H. Polman et al., "Diagnostic criteria for multiple sclerosis: 2010 revisions to the McDonald criteria," Ann. Neurol., Vol.69(2), PP.292-302, 2011.

[6] M. Hutchinson, "Predicting and preventing the future: actively managing multiple sclerosis," Pract. Neurol., Vol.9(3), PP.133-143, 2009.

[7] H. L. Weiner and J. M. Stankiewicz, Multiple sclerosis: diagnosis and therapy. John Wiley \& Sons, Vol.1(5), PP.65, 2012.

[8] K. P. Johnson, "Glatiramer acetate for treatment of relapsing-remitting multiple sclerosis," Expert Rev. Neurother., Vol.12(4), PP.371-384, 2012.

[9] S. Nikfar et al., "Cost-effectiveness of different interferon beta products for relapsing-remitting and secondary progressive multiple sclerosis: Decision analysis based on long-term clinical data and switchable treatments," DARU J. Pharm. Sci., Vol.21(1), P. 50, 2013.

[10] M. Mazdeh, S. Afzali, and M. R. Jaafari, "The therapeutic effect of Avonex, Rebif and Betaferon on EDSS and relapse in multiple sclerosis: a comparative study," Acta Med. Iran., PP.83-88, 2010.

[11] R. Blaşa, A. Huanu, C. Feier, Z. Bajko ${ }^{1}$, and I. Pascu, "Incidence and clinical significance of binding antibodies and their relationships with neutralizing antibodies, both induced by interferon-treatment in multiple sclerosis patients," Rev. Român Med. Lab. Vol, Vol.18(3/4), 2010.

[12] N. Mokhber et al., "Therapeutic effect of A vonex, R ebif and B etaferon on quality of life in multiple sclerosis," Psychiatry Clin. Neurosci., Vol.69(10), PP.649-657, 2015.

[13] N. Madjdinasab, S. E. M. Nejad, S. Tarahomi, F. Sadr, and M. Bahadoram, "Comparison of the Effects of CinnoVex, Rebif and Betaferon on a Expanded Disability Status Scale of Patients With Multiple Sclerosis," Jentashapir J. Heal. Res., Vol.6(3),pp.66-96, 2015.

[14] R. S. Shahtaheri, N. Hatam, and Z. Goudarzi, "Cost-utility analysis of interferon beta-1a (Avonex and Cinnovex) for relapsing remitting multiple sclerosis," J. Pharmacoeconomics Pharm. Manag., Vol.2(3/4), PP.50-54, 2016.

[15] A. Minagar, "Current and future therapies for multiple sclerosis," Scientifica (Cairo)., Vol.2013(2), PP.23-54, 2013. 